A. YAMADA

KODAI MATH. J.

1 (1978), 159-169

\title{
ON THE LINEAR TRANSFORMATIONS OF AHLFORS FUNCTIONS
}

\author{
BY AKIRA YAMADA
}

\section{Introduction.}

Let $R$ be a finite open Riemann surface of genus $\rho$ with $n$ boundary components $\Gamma_{\jmath}(\jmath=0, \cdots, n-1)$. For two distinct fixed points $a, b$ in $R$, it is known that there exist the extremal functions which maximize $|f(b)|$ in the family

$$
\{f ; f \in A(R), f(a)=0,|f| \leqq 1 \text { on } R\}
$$

and they are unique up to a constant multiple of absolute value 1 . The same assertion holds when for a fixed local coordinate we take $\left|f^{\prime}(a)\right|$ instead of $|f(b)|$. They are called Ahlfors functions at $a$ and we denote any of them by $f_{a b}, f_{a}$ respectively. In his lecture note, J. D. Fay [3] has given an explicit representation of the Ahlfors functions of a planar domain by means of theta function. In the present paper, using Fay's result, we obtain a necessary and sufficient condition for the linear transformation of $f_{a b}$

$$
T_{\alpha}\left(f_{a b}\right)(z)=\frac{f_{a b}(z)-f_{a b}(\alpha)}{1-\overline{f_{a b}(\alpha) \cdot f_{a b}(z)}}, \quad \alpha \in R
$$

to be $f_{\alpha_{\beta}}(\beta \in R)$ when $R$ is planar. Let $Q(R)$ denote the set of points $a$ in $R$ such that $T_{\alpha}\left(f_{a}\right)=f_{\alpha}$ for some $\alpha \in R$ distinct from $a$. As a corollary, if $Q(R)$ is not empty, then the double $\hat{R}$ of $R$ is hyperelliptic and $Q(R)$ is the union of $n$ mutually disjoint analytic simple curves in $R$. However in the non-planar case the situation changes. In section 4 we shall show an example of the bordered surface whose $Q(R)$ has non-empty interior. Prof. Suita pointed out to the author that this fact enables us to give a negative answer for the real analyticity of the analytic capacities of non-planar surfaces. But they are real analytic for every plane region $\notin O_{A B}$ [4]. These phenomena show interesting contrasts between non-planar and planar cases. The author wishes to express his sincere thanks to Prof. Suita for his valuable advice and remarks.

\section{Notation}

Fay's notation and definitions [3] will be used in this paper. Let $\phi$ be the

Received November 8, 1976. 
canonical anti-conformal involution of $\hat{R}$, and fix a symmetric canonical homology basis on $\hat{R}$ :

$$
A_{1}, B_{1}, \cdots, A_{\rho}, B_{\rho}, A_{\rho+1}, B_{\rho+1}, \cdots, A_{\rho+n-1}, B_{\rho+n-1}, A_{1^{\prime}}, B_{1^{\prime}}, \cdots, A_{\rho^{\prime}}, B_{\rho^{\prime}},
$$

such that $A_{\rho+k}=\Gamma_{k}$ for $k=1, \cdots, n-1$, and $A_{1}, B_{1}, \cdots, A_{\rho}, B_{\rho}$ (resp. $A_{1^{\prime}}, B_{1^{\prime}}, \cdots$, $\left.A_{\rho^{\prime}}, B_{\rho^{\prime}}\right)$ are cycles in $R$ (resp. $\phi(R)$ ) satisfying the relations in $H_{1}(\hat{R}, \boldsymbol{Z})$ :

$$
\begin{array}{lll}
\phi\left(A_{\imath}\right)=A_{i^{\prime}}, & \phi\left(B_{\imath}\right)=-B_{i^{\prime}}, & 1 \leqq \imath \leqq \rho \\
\phi\left(A_{\imath}\right)=A_{\imath}, & \phi\left(B_{\imath}\right)=-B_{\imath}, & \rho+1 \leqq \imath \leqq \rho+n-1 .
\end{array}
$$

Let $u_{1}, \cdots, u_{g}(g=2 \rho+n-1)$ be a basis of the holomorphic differentials on $\hat{R}$ normalized so that the period matrix with respect to the symmetric canonical homology basis has the form $(2 \pi i I, \tau)$ where $I=$ the identity matrix and $\tau$ is a symmetric matrix with $\operatorname{Re} \tau \leqq 0$. Let $\omega_{b-a}$ be the normalized differential of the third kind on $\hat{R}$ with poles at $b$ of residue 1 and at $a$ of residue $-1, E(x, y)$ the prime form, $\theta(z)=\sum_{m \in \mathbb{Z}} \exp \left(\frac{1}{2}^{t} m \tau m+{ }^{t} m \cdot z\right)$ Riemann's theta function. Then the following symmetries hold [3, Chap. 6]: for $x, y \in \hat{R}, z \in \boldsymbol{C}^{g}$

$$
\omega_{b-a}(x)=\overline{\omega_{b-a}(\bar{x})}, \quad \theta(z)=\overline{\theta(\phi(z))}, \quad E(x, y)=\overline{E(\bar{x}, \bar{y})},
$$

where $\bar{x}=\phi(x)$ the conjugate point of $x$, and for $z_{1}, \cdots, z_{\rho+n-1}, z_{1^{\prime}}, \cdots, z_{\rho^{\prime}} \in \boldsymbol{C}$

$$
\begin{aligned}
\phi\left(z_{1}, \cdots, z_{\rho}, z_{\rho+1}, \cdots, z_{\rho+n-1}, z_{1^{\prime}}, \cdots, z_{\rho^{\prime}}\right) \\
\quad=-\left(\bar{z}_{1^{\prime}}, \cdots, \bar{z}_{\rho^{\prime}}, \bar{z}_{\rho+1}, \cdots, \bar{z}_{\rho+n-1}, \bar{z}_{1}, \cdots, \bar{z}_{\rho}\right) .
\end{aligned}
$$

We denote by $D(f)$ the divisor of a meromorphic function $f$.

\section{Linear transformations of Ahlfors functions.}

Let us write $x-y=\int_{y}^{x} u=\left(\int_{y}^{x} u_{\jmath}\right)_{\jmath=1, \cdots, g}$ for the sake of simplicity. When $R$ is planar $(\rho=0)$, the Ahlfors function can be represented by the theta function and the prime form:

$$
f_{a b}(x)=\varepsilon \cdot \frac{\theta(x-\bar{a}-s) E(x, a)}{\theta(x-a-s) E(x, \bar{a})}, \quad|\varepsilon|=1, \quad s=-\frac{1}{2} \int_{a+\bar{a}}^{b+\bar{b}} u \in \boldsymbol{C}^{g},
$$

where the paths of integation of $s$ must be chosen to be symmetric with respect to $\partial R$ so that $s$ becomes a purely imaginary vector [3, Prop. 6.17].

THEOREM 1. Let $R$ be a plane regular region of finte connectivity. Then the following conditions are equivalent.

(i) $T_{\alpha}\left(f_{a b}\right)=f_{\alpha \beta}$,

(ii) $\frac{1}{2}(a+\bar{a}+b+\bar{b})=\frac{1}{2}(\alpha+\bar{\alpha}+\beta+\bar{\beta})$ in $J_{0}=C^{g} /(2 \pi i I, \tau)$, 
(iii) there exists a meromorphic function $f$ on $R$ satısfying $f(x)>0$ on $\partial R$ and $D(f)=\alpha+\beta-a-b$.

Proof. Clearly we may assume $\varepsilon=1$ in (2) to calculate $T_{\alpha}\left(f_{a b}\right)$. From the symmetries (1),

$$
\begin{aligned}
& T_{\alpha}\left(f_{a b}\right)(x)=\frac{\frac{\theta(x-\bar{a}-s) E(x, a)}{\theta(x-a-s) E(x, \bar{a})}-\frac{\theta(\alpha-\bar{a}-s) E(\alpha, a)}{\theta(\alpha-a-s) E(\alpha, \bar{a})}}{1-\frac{\theta(\alpha-\bar{a}-s) E(\alpha, a)}{\theta(\alpha-a-s) E(\alpha, \bar{a})} \frac{\theta(x-\bar{a}-s) E(x, a)}{\theta(x-a-s) E(\overline{x, \bar{a}})}} \\
& =\eta \cdot \frac{\theta(x-\bar{a}-s) \theta(\alpha-a-s) E(x, a) E(\alpha, \bar{a})-\theta(x-a-s) \theta(\alpha-\bar{a}-s) E(x, \bar{a}) E(\alpha, a)}{\theta(x-a-s) \theta(\bar{\alpha}-\bar{a}-s) E(x, \bar{a}) E(\bar{\alpha}, a)-\theta(x-\bar{a}-s) \theta(\bar{\alpha}-a-s) E(x, a) E(\bar{\alpha}, \bar{a})}
\end{aligned}
$$

where

$$
\eta=\frac{\overline{\theta(\alpha-a-s) E(\alpha, \bar{a})}}{\theta(\alpha-a-s) E(\alpha, \bar{a})}, \quad|\eta|=1 .
$$

This expression can be simplified by the addition-formula of the theta function [3]: for $x, y, a, b \in \hat{R}$ and $e \in \boldsymbol{C}^{g}$,

$$
\begin{gathered}
\theta(x-b-e) \theta(y-a-e) E(x, a) E(y, b)-\theta(x-a-e) \theta(y-b-e) E(x, b) E(y, a) \\
=\theta(e) \theta(x+y-a-b-e) E(x, y) E(a, b) .
\end{gathered}
$$

Disregarding a constant multiple of absolute value 1 , we now have

$$
T_{\alpha}\left(f_{a b}\right)=\frac{\theta(x+\alpha-a-\bar{a}-s) E(x, \alpha)}{\theta(x+\bar{\alpha}-a-\bar{a}-s) E(x, \bar{\alpha})} .
$$

It is obvious that $T_{\alpha}\left(f_{a b}\right)=f_{\alpha \beta}$ if and only if they have the same divisor on $\hat{R}$. By using Riemann's vanishing theorem [3, Th. 1.1] this condition can be expressed as

or

$$
s+a+\bar{a}-\alpha-\bar{\alpha}=\frac{1}{2} \int_{\alpha+\bar{\alpha}}^{\beta+\bar{\beta}} u \quad \text { in } J_{0}
$$

$$
\frac{1}{2}(a+\bar{a}+b+\bar{b})=\frac{1}{2}(\alpha+\bar{\alpha}+\beta+\bar{\beta}) \quad \text { in } J_{0} .
$$

Thus (i) and (ii) are equivalent. On the other hand the equivalence of (ii) and (iii) is a consequence of the next lemma which is a variant of Abel's theorem.

Lemma 1 . Let $R$ be planar. Then

$$
\frac{1}{2}(a+\bar{a}+b+\bar{b})=\frac{1}{2}(\alpha+\bar{\alpha}+\beta+\bar{\beta}) \quad \text { in } J_{0}
$$

if and only if there exists a meromorphuc function $f$ on $R$ with $D(f)=a+b-\alpha-\beta$ satisfying $f>0$ on $\partial R$. 
Proof. Let us introduce the notation $\left\{\begin{array}{l}\delta \\ \varepsilon\end{array}\right\}_{\tau}=2 \pi i \varepsilon+\tau \delta$ for $\varepsilon, \delta \in \boldsymbol{R}^{g}$. Note that for any $x, y \in \hat{R}, x+\bar{x}-y-\bar{y}$ has the form $\left\{\begin{array}{l}0 \\ \varepsilon\end{array}\right\}_{\tau}\left(\varepsilon \in \boldsymbol{R}^{g}\right)$ if we take symmetric paths of integration. Thus the condition $1 / 2(a+\bar{a}+b+\bar{b})=1 / 2(\alpha+\bar{\alpha}+\beta+\bar{\beta})$ in $I_{0}$ is equivalent to

$$
a+\bar{a}+b+\bar{b}=\alpha+\bar{\alpha}+\beta+\bar{\beta}+2\left\{\begin{array}{lll}
0 & \cdots & 0 \\
\nu_{1} & \cdots & \nu_{g}
\end{array}\right\}_{\tau} \quad \text { in } \boldsymbol{C}^{g}, \nu_{j} \in \boldsymbol{Z}(j=1, \cdots, g) .
$$

Assume that this is true, then by Abel's theorem there exists a meromorphic function $f$ on $\hat{R}$ with divisor $D=a+\bar{a}+b+\bar{b}-\alpha-\bar{\alpha}-\beta-\bar{\beta}$. In fact for any fixed $p_{0} \in \Gamma_{0}$ the function

$$
f(p)=\exp \int_{p_{0}}^{p} \omega_{D}, \quad p \in \hat{R}
$$

has the divisor $D$ where $\omega_{D}=\omega_{a-\alpha}+\omega_{\bar{a}-\bar{\alpha}}+\omega_{b-\beta}+\omega_{\bar{b}-\bar{\beta}}$. We must examine the argument of $f$ along $\partial R$. If $p_{j} \in \Gamma_{j}(\jmath=1, \cdots, n-1)$, using the symmetries (1) and Riemann's bilinear relation, we have

$$
\begin{aligned}
\arg f\left(p_{\jmath}\right)=\operatorname{Im} \int_{p_{0}}^{p_{\jmath}} \omega_{D} & =\frac{1}{2 \imath}\left(\int_{-C_{\jmath}} \omega_{D}-\int_{-C_{\jmath}} \bar{\omega}_{D}\right)=\frac{-1}{2 \imath}\left(\int_{C_{\jmath}} \omega_{D}-\int_{\phi^{\prime}\left(C_{j}\right)} \omega_{D}\right) \\
& =-\frac{1}{2 \imath} \int_{B \jmath} \omega_{D}=-\frac{1}{2 \imath} \int_{D} u_{j}=-2 \nu_{j} \pi, \quad \nu_{j} \in \boldsymbol{Z},
\end{aligned}
$$

where $C_{j}$ is a smooth curve in $R$ connecting $p_{j} \in \Gamma_{\text {o with }} p_{0} \in \Gamma_{0}$. Thus $f\left(p_{\jmath}\right)>0$. Similarly, $f(p)>0$ for $p \in \Gamma_{0}$, hence we conclude $f>0$ on $\partial R$.

Next assume that $f$ is a meromorphic function on $R$ with $D(f)=a+b-\alpha-\beta$ satisfying $f>0$ on $\partial R$. Then by reflection with respect to $\partial R, f$ can be extended to $\hat{f}$ which is meromorphic on $\hat{R}$ with $D(\tilde{f})=a+\bar{a}+b+\bar{b}-\alpha-\bar{\alpha}-\beta-\bar{\beta}$. If $B-A$ $(A, B>0)$ is the divisor of a meromorphic function $F$ on $\hat{R}$, by Abel's theorem we have

$$
\int_{A}^{B} u=\left\{\begin{array}{lll}
m_{1} & \cdots & m_{g} \\
n_{1} & \cdots & n_{g}
\end{array}\right\}_{\tau}
$$

where $m_{j}=-\frac{1}{2 \pi \imath} \int_{A_{j}} \mathrm{~d} \log F \in \boldsymbol{Z}$ and $n_{j}=\frac{1}{2 \pi \imath} \int_{B} \mathrm{~d} \log F \in \boldsymbol{Z}(j=1, \cdots, g)$. Therefore $m_{j}=0$ for $D(\tilde{f})$ since $\tilde{f}>0$ on $\partial R$. On the other hand we have

$$
\begin{aligned}
& n_{j}=\frac{1}{2 \pi \imath} \int_{B_{j}} \operatorname{dlog} \tilde{f}=\frac{1}{2 \pi \imath} \int_{C_{j}} \operatorname{dlog} \tilde{f}+\frac{1}{2 \pi \imath} \int_{-\phi\left(C_{j}\right)} \operatorname{dlog} \tilde{f} \\
& =\frac{1}{2 \pi \imath} \int_{C_{j}} \operatorname{dlog} \tilde{f}-\frac{1}{2 \pi \imath} \int_{C_{\jmath}} \overline{\operatorname{dlog} \tilde{f}=} \frac{1}{\pi} \operatorname{Im} \int_{C_{j}} \operatorname{dlog} \tilde{f}
\end{aligned}
$$




$$
=\frac{1}{\pi} \cdot \arg \left(f\left(p_{0}\right) / f\left(p_{\jmath}\right)\right)=2 \nu_{\jmath}, \quad \nu_{j} \in Z \quad \text { q.e. d. }
$$

For example if $R$ is an annulus with center at the origin, the condition of the lemma reduces to the one that $\arg \alpha \beta=\arg a b$.

Letting $b \rightarrow a, \beta \rightarrow \alpha$ in Theorem 1 , we easily obtain

COROLlary. $T_{\alpha}\left(f_{a}\right)=f_{\alpha}$ if and only if $a+\bar{a}=\alpha+\bar{\alpha}$ in $J_{0}$. Moreover assume that the genus of $R \geqq 2$. Then $T_{\alpha}\left(f_{a}\right)=f_{\alpha}$ if and only if $\hat{R}$ is hyperelliptic and $\phi(a)=J(a), \phi(\alpha)=J(\alpha)$ where $J$ is the hyperelliptic involution of $\hat{R}$.

It is natural from this corollary that the next problem is to determine, when $\hat{R}$ is hyperelliptic, the locus of $\phi=J$, or as is the same, the set of fixed points of $\phi \circ J$. Let $h$ be a non-trivial automorphism of $\hat{R}$ and $H$ be the set of fixed points of $h$.

LEMMA 2. If $h \circ \phi=\phi \circ h$, then either $H \subset \partial R$ or $H \cap \partial R=\phi$ (empty set). In the second case, for any $p \in H \cap R$

$$
H \cap R \subset\left\{x \in R ; f_{p}(x)=0\right\} .
$$

Proof. If $H \subset \partial R$, there is nothing to prove. Thus we may assume $H \backslash \partial R$ $\neq \phi$. It is then easily verified from the hypothesis of the lemma that $h(R)=R$ and $h$ is an automorphism of $R$. Fix $p \in H \cap R$ and consider the function $f_{p} \circ$.

$$
f_{p} \circ h(p)=0, \quad\left|f_{p} \circ h\right| \leqq 1 \quad \text { on } R
$$

For a suitable choice of the local coordinate $z$ centered at $p, h$ has the form $h(z)=\varepsilon z\left(\varepsilon^{N}=1, \varepsilon \neq 1\right)$ in a neighborhood of $p$. Taking the derivative of $f_{p} \circ h$ at $p$, we have $\left(f_{p} \circ h\right)^{\prime}(p)=\varepsilon f_{p}^{\prime}(p)$. Hence $f_{p} \circ h$ is an Ahlfors function at $p$ and $f_{p} \circ h(x)=\varepsilon f_{p}(x)$ for all $x$ in $R \cup \partial R$, since Ahlfors functions are unique. Suppose $x \in H \cap(R \cup \partial R)$, then $f_{p}(x)=\varepsilon f_{p}(x)$, so that $f_{p}(x)=0$. This means that $x \in R$ which gives $H \cap \partial R=\phi$.

q.e.d.

Let $\hat{R}$ be hyperelliptic and $h=J$ its hyperelliptic involution. Since $J \circ \phi=\phi \circ J$, it follows from lemma 2 that $W \subset \partial R$ or $W \cap \partial R=\phi$, where $W$ is the set of all Weierstrass points. More precisely we have

THEOREM 2. Let the double $\hat{R}$ of $R$ be hyperelliptic of genus $g$.

(i) Assume $\rho=0: W$ is contained in $\partial R$ and there are two Welerstrass points on each $\Gamma_{\mathrm{J}}(j=0, \cdots, n-1)$. The locus of $\phi=J$ is a union of $n$ mutually disjoint analytic simple closed curves in $R$ passing through the Wererstrass points on $\partial R$.

(ii) Assume $\rho>0$ : The number of the boundary components of $R$ is one or two, and $W \cap \partial R=\phi$. The Ahlfors functions at the $g+1$ Weierstrass points in $R$ are identical and have $g+1$ zeros at these Weierstrass points. The locus of $\phi=J$ is empty.

Proof. First we consider the case where $W \subset \partial R$. Since $\hat{R}$ is hyperelliptic, 
$\hat{R}$ has $2 g+2$ Weierstrass points. From $2 g+2 \geqq 2 n$, we see that one of the boundary components of $R$, say $\Gamma_{0}$, contains at least two Weierstrass points. If $W_{1}, W_{2} \in \Gamma_{0}$ are those Weierstrass points, then from the hypothesis there exists a meromorphic function $w(x)$ on $\hat{R}$ with $D(w)=2 W_{1}-2 W_{2}$. Comparison of the divisors gives

$$
w(\phi(x))=\lambda \cdot \overline{w(x)}, \quad \lambda \in \boldsymbol{C},|\lambda|=1, x \in \hat{R} .
$$

For a suitable $\varepsilon \in \boldsymbol{C}, w_{0}(x)=\varepsilon \cdot w(x)$ satisfies

$$
w_{0}(\phi(x))=\overline{w_{0}(x)} \text {. }
$$

Therefore $w_{0}\left(\Gamma_{j}\right)(\jmath=0, \cdots, n-1)$ is a continuum in $\boldsymbol{R} \cup\{\infty\}$ so that a closed interval in $\boldsymbol{R} \cup\{\infty\}$. It is easy to see that each $\Gamma_{\text {o covers }} w_{0}\left(\Gamma_{\text {J }}\right)$ exactly twice. Since $w_{0}$ is of order 2 , we have

$$
w_{0}\left(\Gamma_{\jmath}\right) \cap w_{0}\left(\Gamma_{k}\right)=\phi \quad(\jmath \neq k) .
$$

From the argument principle $w_{0}$ maps $R$ conformally onto a slit region $w_{0}(R)$

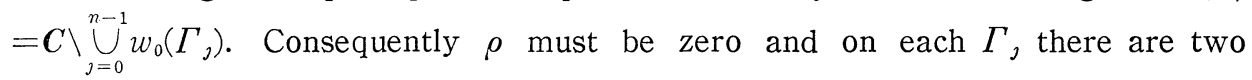
Weierstrass points which correspond to the end points of $w_{0}\left(\Gamma_{\jmath}\right)$. To determine the locus of $\phi=J$ we claim that

$$
\{x \in \hat{R} ; \phi(x)=J(x)\}=W \cup\left\{x \in \hat{R} ; w_{0}(x) \in \boldsymbol{R}, x \notin \partial R\right\} .
$$

This follows easily from the functional equation $\overline{w_{0}(x)}=w_{0}(\phi \circ J(x))$. Now it is clear that the locus consists of $n$ disjoint simple closed analytic curves passing through the Weierstrass points. We will then be finished with the proof of (i) if we can show $\rho>0$ whenever $W \cap \partial R=\phi$.

Suppose that $W \cap \partial R=\phi$. Let $Q$ be a Weierstrass point in $R$. Then there exists, as in the proof of the first part, a mermorphic function $w_{1}$ on $\hat{R}$ with $D\left(w_{1}\right)=2 Q-2 \phi(Q)$ satisfying

$$
w_{1}\left(\phi(x) \overline{w_{1}(x)}=1 .\right.
$$

The restriction of $w_{1}$ to $R$ is a unitary function on $R$ having only a double zero at $Q \in R$. But on bordered surfaces every non-constant unitary holomorphic function has at least $n$ zeros, where $n$ is the number of the boundary components. Thus $n$ must be one or two. The hyperellipticity of $\hat{R}$ implies that $g=2 \rho+n-1$ $\geqq 2$. From $n \leqq 2$ we conclude that $\rho>0$. This completes the proof of (i) and the first part of (ii).

Next we proceed to prove the rest of (ii). Let $p \in W \cap R$. Then by Lemma 2 we have

$$
W \cap R \subset\left\{x \in R ; f_{p}(x)=0\right\} .
$$

But it is known that the Ahlfors function has at most $g+1$ zeros [1]. Noting 
that $W \cap R$ consists of $g+1$ points, we have indeed

$$
W \cap R=\left\{x \in R ; f_{p}(x)=0\right\} .
$$

Thus the Ahlfors functions at the Weierstrass points in $R$ are 1dentical with each other, since they have the same divisor. To complete the proof we have to show that the locus of $\phi=J$ is empty. Assume that $\phi(x)=J(x)$ for $x \in \hat{R}$. Then we obtain

$$
1=w_{1}\left(\phi(x) \overline{w_{1}(x)}=w_{1}(J(x)) \overline{w_{1}(x)}=\left|w_{1}(x)\right|^{2} .\right.
$$

Hence $x \in \partial R$ and so $x=J(x)$ or $x \in W$, contradicting the fact that $W \cap \partial R=\phi$.

The proof of Theorem 2 contains the following: every regular plane region whose double is hyperelliptic is conformally equivalent to a region slit along a finite number of segments on a line. The converse is clearly also true.

\section{Example.}

Let $D_{1}$ and $D_{2}$ be two copies of a closed unit disk with slits along the segments $[s, t],[-t,-s](0<s<t<1)$. We construct the desired finite bordered surface $S$ by joining $D_{2}$ to $D_{1}$ along their common distinguished slits in the standard manner (i.e. the upper edge of such a slit of a given copy being joined to the lower edge of the corresponding slit of the other copy). The double $\hat{S}$ of $S$ can be expressed explicitly by the algebraic equation

$$
y^{2}=\left(x^{2}-s^{2}\right)\left(x^{2}-t^{2}\right)\left(x^{-2}-s^{2}\right)\left(x^{-2}-t^{2}\right) .
$$

Thus $\hat{S}$ is hyperellitic of genus 3 . It follows that $\phi(x, y)=((1 / \bar{x}), \bar{y})$ is the canonical anti-conformal involution of $\hat{S}$ and that $S=\{(x, y) \in \hat{S} ;|x| \leqq 1\}$. Denote by $O_{1}, O_{2}$ (resp. $\infty_{1}, \infty_{2}$ ) the points of $\hat{S}$ lying over the origin (resp. the point at infinity) in such a way that we have

$$
x^{2} y=s t+O\left(x^{2}\right), \quad x^{2} y=-s t+O\left(x^{2}\right), \quad x^{-2} y=s t+O\left(x^{-2}\right), \quad x^{-2} y=-s t+O\left(x^{-2}\right)
$$

near these points, respectively. In this section we shall show that the Ahlfors function for $S$ at $p$ in a sufficiently small neighborhood of $O_{1}, O_{2}$ has the form

$$
\varepsilon \cdot \frac{x-p}{1-\bar{p} x}, \quad|\varepsilon|=1 \text {. }
$$

Consequently, $S$ is an example of the bordered surface whose $Q(S)$ has nonempty interior.

To show this we begin by studying the meromorphic differential $\psi$ on $S$ with $D(\psi) \geqq O_{2}-O_{1}+\infty_{2}-\infty_{1}$. Such differentials, by the Riemann-Roch theorem, form a 2-dimentional vector space. Indeed, easy calculation shows that its basis is given by 


$$
\frac{d x}{x y}, \frac{\left\{y+s t\left(x-x^{-1}\right)^{2}\right\} d x}{x y} .
$$

The former is holomorphic and the latter is meromorphic with simple poles at $O_{1}, \infty_{1}$.

Lemma 3. Let $\phi=\frac{\left\{y+s t\left(x-x^{-1}\right)^{2}+\lambda\right\} d x}{\imath x y}, \lambda \in C$. Then $\phi$ is a positive differential if and only if

$$
-\left(1-s^{2}\right)\left(1-t^{2}\right) \leqq \lambda \leqq\left(1-s^{2}\right)\left(1-t^{2}\right) .
$$

Proof. By definition the positiveness of $\phi$ means that

$$
\frac{y+\operatorname{st}\left(x-x^{-1}\right)^{2}+\lambda}{y} \geqq 0 \quad \text { for all }|x|=1 .
$$

Since $y$ is real for $|x|=1, \lambda$ must also be real. Since $\pm y$ correspond to a fixed $x$, we have

$$
\pm \frac{s t\left(x-x^{-1}\right)^{2}+\lambda}{y} \geqq-1 \quad \text { for all }|x|=1
$$

or

$$
\left\{s t\left(x-x^{-1}\right)^{2}+\lambda\right\}^{2} \leqq y^{2} \quad \text { for all }|x|=1 .
$$

Set $r=x^{2}+x^{-2}$ for convenience, then from (3) we can replace the above inequality by

$$
\{s t(r-2)+\lambda\}^{2} \leqq\left(1+s^{4}-s^{2} r\right)\left(1+t^{4}-t^{2} r\right), \quad-2 \leqq r \leqq 2 .
$$

This inequality is linear with respect to $r$, and so setting $r= \pm 2$, we obtain

$$
\lambda^{2} \leqq\left(1-s^{2}\right)^{2}\left(1-t^{2}\right)^{2}, \quad(\lambda-4 s t)^{2} \leqq\left(1+s^{2}\right)^{2}\left(1+t^{2}\right)^{2} .
$$

These inequalities yield (5).

q.e.d.

Next let $W$ be the family of all differentials $\omega$ holomorphic on $S \cup \partial S$ except at $a \in S$ where $\omega$ has a principal part

$$
\frac{d z(x)}{(z(x)-z(a))^{2}}+\frac{\eta \cdot d z(x)}{z(x)-z(a)}, \quad \eta \in \boldsymbol{C}
$$

for a fixed local coordinate $z$ around $a \in S$. Following Ahlfors [1], for any $a \in S$ there exists an extremal differential minimizing the expression $\int_{\partial S}|\omega|$ in $W$. It is in general not unique. But if we denote any of it by $\omega_{a}$ and set $\psi_{a}=f_{a} \omega_{a}$, then it is known to be characterized by $\arg \psi_{a}=$ const. on $\partial S$. This characterization and the fact that $\left|f_{a}\right|=1$ on $\partial S$ imply that $f_{a}, \omega_{a}, \psi_{a}$ are extended meromorphically across $\partial S$ to $\hat{S}$. Setting $D\left(f_{a}\right)=a+A_{a}$ on $S$ we obtain 
(6)

$$
\begin{array}{ll}
D\left(f_{a}\right)=a+A_{a}-\bar{a}-\bar{A}_{a} & \\
D\left(\omega_{a}\right)=B_{a}-2 a+2 \bar{A}_{a}+\bar{B}_{a} & \text { on } \hat{S} \\
D\left(\psi_{a}\right)=A_{a}+B_{a}-a+\bar{A}_{a}+\bar{B}_{a}-\bar{a} &
\end{array}
$$

where $B_{a}$ is a positive divisor satisfying $a \notin\left\{B_{a}\right\} \subset S \cup \partial S$. Conversely, if $f$ is a unitary function on $\hat{S}$ and $\psi$ a positive differential on $\hat{S}$ with $D(f)$ and $D(\phi)$ having the form (6) for some divisors $A$ and $B$, then $f$ is an Ahlfors function at $a$. Since the degree of a meromorphic differential is $2 g-2$, we have

$$
\operatorname{deg} A_{a}+\operatorname{deg} B_{a}=g \text {. }
$$

Set $N(a)=1+\operatorname{deg} A_{a}$ (the number of the zeros of $f_{a}$ ) and call any extremal differential $\omega_{a}$ a conjugate differential of $f_{a}$.

LEMMA 4. If there exists a conjugate differential $\omega_{a}$ having no zeros on $\partial S$, then $N(p)=N(a)$ for $p$ in a neighborhood of $a$.

Proof. It is easily verified from Hurwitz's theorem and a normal family argument that $N(p)$ is lower semi-continuous, i. e.,

$$
N(a) \leqq N(p), \quad p \in U_{1}
$$

in some neighborhood $U_{1}$ of $a$. We shall show under the hypothesis of Lemma 4 that $N(p)$ is also upper semi-continuous. To this end we proceed as follows.

Since $\omega_{a}$ is a Schottky differential with only a double pole at $a$, the residue of $\omega_{a}$ at a vanishes. Therefore if we set

$$
\omega_{p}(x)=\pi L(x, p)+\Omega_{p}(x)
$$

for $x, p \in \hat{S}$ where $L(x, p)$ is the adjoint Bergman kernel [2] and $\Omega_{p}(x)$ is a holomorphic differential on $\hat{S}$, then we obtain

$$
\begin{aligned}
\frac{1}{2 \pi} \int_{t \in \partial S}\left|\Omega_{p}(t)\right| & \leqq \frac{1}{2 \pi} \int_{t \in \partial S}\left|\omega_{p}(t)\right|+\frac{1}{2} \int_{t \in \partial S}|L(t, p)| \\
& =\left|f_{p}{ }^{\prime}(p)\right|+\frac{1}{2} \int_{t \in \partial S}|L(t, p)| .
\end{aligned}
$$

Note that the last equality holds by the duality relation between $f_{p}$ and $\omega_{p}[1]$. It is clear that the last expression is bounded for $p \in U_{1}$, so that

$$
\frac{1}{2 \pi} \int_{t \in \partial S}\left|\Omega_{p}(t)\right| \leqq K, \quad \text { for } p \in U_{1}
$$

with finite $K$. Let $U_{2}$ be a sufficiently small neighborhood of a satisfying $\bar{U}_{2} \subset$ $U_{1} \backslash\left\{B_{a}\right\}$. This is possible because $a \notin\left\{B_{a}\right\}$. Fix a nowhere-vanishing holomorphic differential $\xi(x)$ on $S \cup \partial S$. Then clearly $\frac{L(x, p)}{\xi(x)}$ is locally bounded in $S \backslash \bar{U}_{2}$ 
for $p \in U_{2}$. On the other hand, we obtain from Green's formula

$$
\begin{gathered}
\left|\frac{\Omega_{P}(x)}{\xi(x)}\right|=\frac{1}{2 \pi}\left|\int_{t \in \partial S} \frac{\Omega_{p}(t)}{\xi(t)} d G_{x}(t)\right| \leqq \frac{1}{2 \pi} \operatorname{Max}_{t \in \partial S}\left|\frac{d G_{x}(t)}{\xi(t)}\right| \cdot \int_{t \in \partial S}\left|\Omega_{p}(t)\right| \\
\leqq K \cdot \operatorname{Max}_{t \in \partial S}\left|\frac{d G_{x}(t)}{\xi(t)}\right|, \quad p \in U_{2},
\end{gathered}
$$

where $d G_{x}(p)=d g(p, x)+i^{*} d g(p, x), g$ being the Green's function of $S$. Thus the family $\left\{\omega_{p}(x) / \xi(x)\right\}_{p \in U_{2}}$ is locally bounded in $S \backslash \bar{U}_{2}$, so that it is normal there. Since $\left\{B_{a}\right\} \subset S \backslash \bar{U}_{2}$, a similar reasoning as in the case of Ahlfors functions gives that $\operatorname{deg} B_{a}$ is also lower semi-continuous at $a$, i. e., there is a neighborhood $U\left(\subset U_{2}\right)$ of a such that

$$
\operatorname{deg} B_{a} \leqq \operatorname{deg} B_{p}, \quad p \in U .
$$

From (7), (8), (9) one obtains the desired result.

q.e.d.

Now it is almost clear how to prove the before mentioned assertion concerning our example. By Lemma 3 and the remark stated below (6), $x$ is an Ahlfors function at $O_{1}$ since $D(x)=O_{1}+O_{2}-\infty_{1}-\infty_{2}$ in $\hat{S}$. Thus $N\left(O_{1}\right)=2$. But the proof of Lemma 3 shows that $\psi>0$ on $\partial S$ if $-\left(1-s^{2}\right)\left(1-t^{2}\right)<\lambda<\left(1-s^{2}\right)\left(1-t^{2}\right)$. Hence by Lemma $4 N(p)=2$ in some neighborhood $U$ of $O_{1}$, so that $f_{p}(p \in U)$ is a fractional linear transformation of $x$. Since $\left|f_{p}\right|=1$ on $\partial S, f_{p}$ must have the form

$$
\varepsilon \cdot \frac{x-p}{1-\bar{p} x}, \quad|\varepsilon|=1, p \in U .
$$

By symmetry the same assertion holds for $\mathrm{O}_{2}$.

It is of some interest to see that at $\pm s, \pm t$, the Weierstrass points in $S$, the Ahlfors functions are given by

$$
\varepsilon \cdot \frac{y}{x^{2}\left(x^{-2}-s^{2}\right)\left(x^{-2}-t^{2}\right)}=\varepsilon \cdot \sqrt{\frac{\left(x^{2}-s^{2}\right)\left(x^{2}-t^{2}\right)}{\left(1-s^{2} x^{2}\right)\left(1-t^{2} x^{2}\right)}}, \quad|\varepsilon|=1 .
$$

This is a direct consequence of part (ii) of Theorem 2. Thus $N(p)=4$ in some neighborhoods of $\pm s, \pm t$. Furthermore, a closer examination using the method of this paper will show that $N(p)=4$ also in a neighborhood of $\partial S$.

Finally we remark that the analytic capacity $C_{B}(z)$ is not real analytic on $S$. The Gaussian curvature of $C_{B}$ is given by

$$
-\frac{1}{C_{B}{ }^{2}} \cdot \frac{\partial^{2} \log C_{B}(z)}{\partial z \partial \bar{z}}
$$

and is constant -1 in some neighborhood of $O_{1} \in S$ since there we have

$$
C_{B}(z)=\frac{\left|f_{o_{1}}{ }^{\prime}(z)\right||d z|}{1-\left|f_{o_{1}}(z)\right|^{2}} .
$$


If $C_{B}$ is real analytic on $S$, then the curvature is -1 everywhere on $S$ by analytic continuation. However, when compaired with the Poincaré metric by using Ahlfors' version of Schwarz's lemma, this gives rise to a contradiction.

\section{REFERENCES}

[1] Ahlfors, L., Open Riemann surfaces and extremal problems on compact subregions, Comm. Math. Helv., Vol. 24 (1950), 100-134.

[2] Bergman, S., The kernel function and conformal mapping, Math. Surveys 5, Amer. Math. Soc. Providence, R. I., 1950.

[3] FAY, J.D., Theta functions on Riemann surfaces, Springer-Verlag, Lecture Notes, Vol. 352, 1973.

[4] Suita, N., On a metric induced by analytic capacity, Kōdaı Math. Sem. Rep. 25 (1973), 215-218.

Department of Mathematics,

Tokyo Institute of Technology,

OH-OKayama, Meguro-Ku,

TOKYO, JAPAN 
